\title{
THE ROLE OF SKETCHING ACTIVITIES AND OUTCOMES IN CONCEPTUAL DESIGN PHASE
}

\author{
Nikolić, Marija (1); Škec, Stanko (1,2); Martinec, Tomislav (1); Horvat, Nikola (1) \\ 1: University of Zagreb; 2: Technical University of Denmark (DTU)
}

\begin{abstract}
Sketching-related activities are considered as an essential form of communication in the early phases of a design process. In the presented study, it is argued that both the sketching and the sketch-related verbalisations are reflected in the level of elaboration of the sketching outputs. Hence, a protocol study was conducted to analyse the frequencies of different sketching-related activities during team conceptual design sessions and the associated levels of elaboration for each of the sketching outputs in the form of concept drawings. The results show that although teams generate sketches of various number, complexity and clarity, there exist commonalities across the studied experiment sessions. For example, teams share a pattern of developing solutions without transformations or using lateral transformations within the first part of the sessions and using vertical transformations to produce final concepts towards the end of the sessions. Moreover, teams used associated sketch elements to start drawing new sketches and then alternated to other activities, most of all verbal explanation, for the sake of elaboration and better understanding.
\end{abstract}

Keywords: Sketching, Teamwork, Conceptual design, Protocol analysis, Communication

\section{Contact:}

Martinec, Tomislav

University of Zagreb, FSB

Department of Design

Croatia

tomislav.martinec@fsb.hr

Cite this article: Nikolić, M., Škec, S., Martinec, T., Horvat, N. (2019) 'The Role of Sketching Activities and Outcomes in Conceptual Design Phase', in Proceedings of the 22nd International Conference on Engineering Design (ICED19), Delft, The Netherlands, 5-8 August 2019. DOI:10.1017/dsi.2019.43 


\section{INTRODUCTION}

Collaboration in design is characterised by the elements of both verbal and non-verbal communication (Mc Neill et al., 1998). Sketching has been considered as an essential tool for non-verbal communication within design teams in the early phases of a design process (Fish and Scrivener 1990), whereas studies have shown that designers employ sketching to improve the understanding and framing of a given design problem, but also to facilitate the creation and analysis of solution attempts (van der Lugt, 2002). It is a common belief that designers utilise sketching as a support for their thinking processes. Sketches can, for example, reduce cognitive load by providing an extension to the designer's memory (Purcell and Gero 1998). Hence, sketching, as a means of graphical and textual communication, can in that way facilitate the development of a shared mental model of the design problem and lead to an improved shared understanding of the proposed solutions.

In addition to sketching, the verbal communication, such as the questioning and clarification of concepts, represents an important mode of communication in design teams throughout the conceptual design phase, as it leads to better understanding and elaboration of concept solutions (Eris et al., 2014). On the other hand, sketching helps designers to elaborate the concepts by enabling the visualisation of thoughts that would be almost impossible to externalise solely through verbalisation. It can thus be argued that both the sketching and the sketch-related verbalisations are reflected in the level of elaboration of the sketching outputs. Moreover, while sketching of the design content from scratch facilitates identification of new features and relations associated among design concepts (Sachse et al., 2004), the verbal communication helps in transferring, gaining and exchanging of knowledge among design team members (Ariff et al., 2012). The interaction between sketching and verbal communication can be perceived as an essential communication medium in the early stages of the design process, where designers support their verbal argumentation with visual representations and vice versa.

Here presented work is an exploratory step towards the understanding how design teams perform sketching-related activities (both verbal and non-verbal) and how they elaborate the associated sketches when executing conceptual design tasks. Two main research questions that guided the study are:

- What are the frequencies of various sketching-related activities in team conceptual design sessions?

- In what way is sketching used for sketch elaboration during team conceptual design sessions?

In order to tackle the research questions, the presented study was conducted by employing the protocol analysis method. The coding schemes and measures for analysing sketching-related activities and the level of elaboration of sketching outputs have been developed based on the studies of sketching and methods of sketch evaluation in conceptual design, which are introduced within Section 2. By selecting and adapting specific coding schemes, the frequencies of different types of sketches and sketching-related activities have been analysed. The overview of the empirical part of the study is presented in Section 3. Finally, once the sketching outputs were analysed and the session protocols were coded, it was determined which measurements are associated with which types of sketching-related activities. These insights together with the discussion of the empirical study results are given in Section 4.

\section{BACKGROUND}

\subsection{Sketching in conceptual design}

In the conceptual design phase, designers mainly use freehand sketches to support forming of creative ideas (Suwa et al., 1998) and to explore their thoughts, reflect on their work and express design thinking (Agogino et al., 2006). In this manner, when performing sketching, designers are more likely to discover new features and relations which can then be used to refine and revise existing ideas. Designers are therefore encouraged to draw repeatedly in order to develop new solutions.

Many scholars have analysed how designers use sketches during the early stages of the design process (Goldschmidt, 1991; Goel, 1995; Suwa et al., 1998). For decades, researchers have employed experimental studies to investigate sketching as a set of cognitive activities (see, e.g. Ullman et al., 1990; Ariff et al., 2012). These studies have shown that sketching is an important medium of communication when it comes to transferring ideas and mental models of the design problem from one member of the team to the others. Ullman et al. (1990) defined sketching as a cognitive process in a way that "designers present the information internally in their thoughts and externally on paper, computer screen or other media." Based on the existing understanding, while sketching, the designer initially develops a mental model of the design 
problem. Afterwards, this mental model is altered and refined using the insights that have been gained through exploration of uncertainties and contradictions associated with a given design problem (Scrivener et al., 2000). Such a refined mental model of the design problem can then result in sketching activity and serve as a medium for communication and information exchange within the design team. Therefore, during team design sessions, shared sketches provide an underlying basis for both the shared attention and shared understanding (Heiser et al., 2004).

Some of the empirically developed models of sketching describe the way sketching is carried out. For example, Ariff et al. (2012) modelled the effects of sketching on the interpretation and reinterpretation of the mental model of the design problem and the knowledge gain through communication. Consequently, sketching is described as the processing of three main cognitive patterns (Ariff et al., 2012). The first pattern reveals that the mental model of the design problem stimulates questions, answers and instructions on what to explore and how to get a revised mental model of the problem for further development of solutions through cycles of research, interpretation and reinterpretation. The second pattern implies that by defining uncertainties and ambiguities, there is an opportunity for creative interventions, although uncertainties and fuzziness can limit the completeness of the mental model of the design problem. Finally, the third pattern indicates that individual thinking processes and communication cycles in design teams both alternate between gaining, transferring and exchanging knowledge. Ariff et al. (2012) developed a coding scheme based on these three patterns and used it to explore how different communication mediums are used during sketching-related activities.

\subsection{Protocol analysis of sketching-related activities}

The majority of the sketching-related experimental research has been conducted using the protocol analysis method which, according to Gero (1990), is the most appropriate method for cognitive processes analysis. The protocol analysis method allows conversion of verbal statements into segmented data for analysis and has as such been intensively used within the field of design research (Suwa et al., 1998). The method is used for either one of two distinctive types of design studies: process-oriented and content-oriented (Dorst \& Dijkhuis, 1995). The first focuses on describing the structure of the design process in the context of general problem-solving taxonomy, such as plans, strategies, goals, etc., and the latter discloses the content of information, resources and knowledge that are used to conclude. Moreover, two different techniques of protocol analysis can be described as "think aloud" and "retrospective report" approaches (Suwa et al., 1998). In the former, the subjects are asked to talk aloud about their thoughts while working on the given task, while in later technique the subjects are asked to remember and report their past thoughts after the task.

Within the sketch-related research, the protocol analysis is most often performed as a "think aloud" method, with a focus both on the process and the content of sketching. Furthermore, these studies are primarily tied to the conceptual design phase; hence protocol analysis is primarily employed as a means of coding and analysing data related to concept sketches and the process of their creation. For example, Suwa et al. (1998) used a coding scheme consisting of 4 categories (physical, perceptual, functional and conceptual) to classify the contents of the designer's thoughts or action. On the other hand, the goal of a study by Goldschmidt (1991) was to isolate sketching activities and use visual thinking and images as a conceptual framework for the research. The study introduces an explanation for the terms of motion, the arguments by which the protocol would be segmented and the corresponding coding scheme. The coding scheme consists of two modalities that are attached to each argument: (1) "seeing as" which signifies figurative arguments during sketching and (2) "seeing that" which indicates an improvement of nonfigurative arguments relating to the subject that is being created. Ariff et al. (2012) proposed the DesignCommunication Block Framework, which categorises sketch elements based on the sketch types given by Ullman et al. (1990) to support notation and graphic representation. Moreover, they associated sketches and their elements with the following four main categories of sketching activities: drawing is an activity described by introducing basic graphic forms and function elements, detailing is characterized by articulation of sketch elements from drawing, explaining enables communication of elemental meanings from drawing categories or detailing with graphical or numerical notes and transferring is an activity that communicates the meaning of elements in the drawing or detailing category with textual notes. Due to the detailed description and categorisation of sketch elements, the coding scheme of the DesignCommunication Block Framework (Ariff et al., 2012) will be used in this paper with some minor changes. Therefore, to better understand sketching, it is necessary to monitor the development of sketches and their elements as outputs of the abovementioned sketching activities. 


\subsection{Analysis of sketch elaboration}

The level to which the sketches generated as part of experimental design research are elaborated have been assessed using various evaluation methods. Here, elaboration represents the process of embellishing an idea by adding details that encourage designers to expand their ideas and organise their thinking, as well as helping them to clarify and articulate their thoughts (Sevier et al., 2017). Rodgers et al. (2000) focused on the amount of information provided in a sketch, indicated explicitly by the sketch attributes, such as shading. In contrast, Besemer (1998) introduced elaboration and synthesis as degrees to which a particular sketch integrates different elements into a coherent whole. Another approach was proposed by Kudrowitz et al. (2012), who probed the quality of sketches by addressing the clarity facet of elaboration based upon overall line quality and correctness of perspective and proportion. Moreover, Goel (1995) introduced vertical transformation in design sketches where more detail and clarification of neater lines are observed, while Chen et al. (2003) have developed a complexity scale to calculate the degree of transformation. By using complexity metrics, they could statistically demonstrate the relation between the number and the complexity of the sketches. Finally, Dean et al. (2006) emphasised specificity to describe not only the level of detail, but also the clarity of a sketched idea.

Although the studies mentioned above investigated different aspects of sketch elaboration, the majority did not measure elaboration directly, as this aspect was instead combined with other constructs. Sevier et al. (2017) addressed this issue by developing a method of elaboration assessment based on a link between the quantity (complexity) and quality of information (clarity).

In the presented study, the analysis and evaluation of the sketches have been used to monitor the concept development throughout team conceptual design sessions and to draw conclusions of how the level of elaboration of the proposed concept sketches depends on the number and order of sketches generated. Furthermore, by using the sketch evaluation methods, the relationship between activities and sketches has been studied according to the criteria as mentioned above.

\section{EMPIRICAL STUDY}

\subsection{Experimental research, participants and design task}

The sketches and the sketch-related activities have been investigated by performing a protocol analysis of five teams solving a conceptual design task during the 60-minute experimental sessions. The recordings from these sessions were segmented, coded and analysed to provide the results appropriate for addressing the research questions. Experiment participants were selected from the third and fourth-year mechanical engineering students. They gained experience in concept design and general expertise in the design and development of products through participation in project-based courses which included planning, conceptual and detail design, and prototype assembly and testing. Additionally, the students have been enrolled in courses such as Product Development, Design Methods and Advanced Computer-Aided Design (CAD). The similar background of the experiment participants enabled the forming of teams with similar levels of knowledge. Prior to the experiment sessions, the participants' problem-solving style has been assessed (see Martinec et al., 2018 for more details). Fifteen participants were then randomly grouped into five teams of three. The arrangement of equipment in the room where the sessions took place was strictly defined. The room was equipped with two video recording cameras, one voice recorder and an electronic writing block for sketching. The task brief was displayed on a screen mounted on the wall. The screen also displayed information on time left until the end of the session. In short, the task was to come up with a concept of a portable key-organising solution to help users deal with everyday key usage. Because the keys are a standard object of everyday life, it was considered that the design brief should not be introduced to the teams prior to the experiment sessions and that no literature would be required to solve the task. The concepts should have been supported with sketches, including the approximate dimensions, materials selection and usage scenarios. Participants were encouraged to work on the development of multiple concepts but to indicate the solution which they selected to be further developed.

\subsection{Sketching outputs analysis}

The developed concepts were analysed using the elaboration metrics proposed by Sevier et al. (2017) to obtain insights about complexity and clarity values for different sketches. The complexity from the bottomup approach is defined with the help of eight criteria: shading, three-dimensionality, annotations, scale, alternative view/configuration, variable line weight, kinetics and texture, and clarity based on top-down 
approach with six criteria: who, what, when, where, why and how. The used set of criteria guided an objective and systematic assessment of developed concepts and enabled the analysis of the extent to which various concept sketches fulfilled and balanced these complexity and clarity criteria throughout the session. The detailed descriptions of the measures and the procedures for assessing the complexity and clarity of sketches are available in Sevier et al. (2017).

In addition to the complexity and clarity levels, the process of sketch generation and elaboration can be analysed based on the moves between sketches (Yuan et al., 2018). As mentioned earlier, one of the methods by which concept elaboration can be analysed is the transformation method developed by Goel (1995) who defined two types of processes that take place between successive sketches in the conceptual stage of product development. Goel (1995) defines lateral transformation as the transformation that changes the concept into another, but the completely different concept (as opposed to a more detailed version of the same concept, unrelated or identical concept). Generally, lateral transformation takes place when a new concept is drawn up that is based on the principle of the already drawn, existing concept, but there are visible differences between them. The other type of transformation introduced by Goel (1995) is the vertical transformation, which upgrades the existing concept through explaining and detailing. In general, vertical transformation takes place when one concept is developed by means of detail, and this more detailed concept, apart from of course the added details, does not differ from the previous concept in the context of function and form. Yuan et al. (2018) explain that transformations can result from three types of associations - shape, behaviour and function - and that designers can be guided to perform certain transformation actions and generate diverse sketches of high quality.

Within the presented empirical study, the complexity, clarity and sketch transformations have all been analysed on a concept level, where each concept consists of one or more associated sketches. Complexity and clarity were analysed based on the individual concepts, whereas the transformations were analysed for the pairs of concepts. Hence, for each concept generated by the team, the number of satisfied complexity and clarity criteria has been recorded, and for each combination of two different concepts, the type of transformation has been determined. In cases where neither the lateral or the vertical transformation exists between two consecutively generated concepts, a move without the transformation was recorded. An example of analysing the complexity, clarity and transformation between two concept sketches is shown in Figure 1.

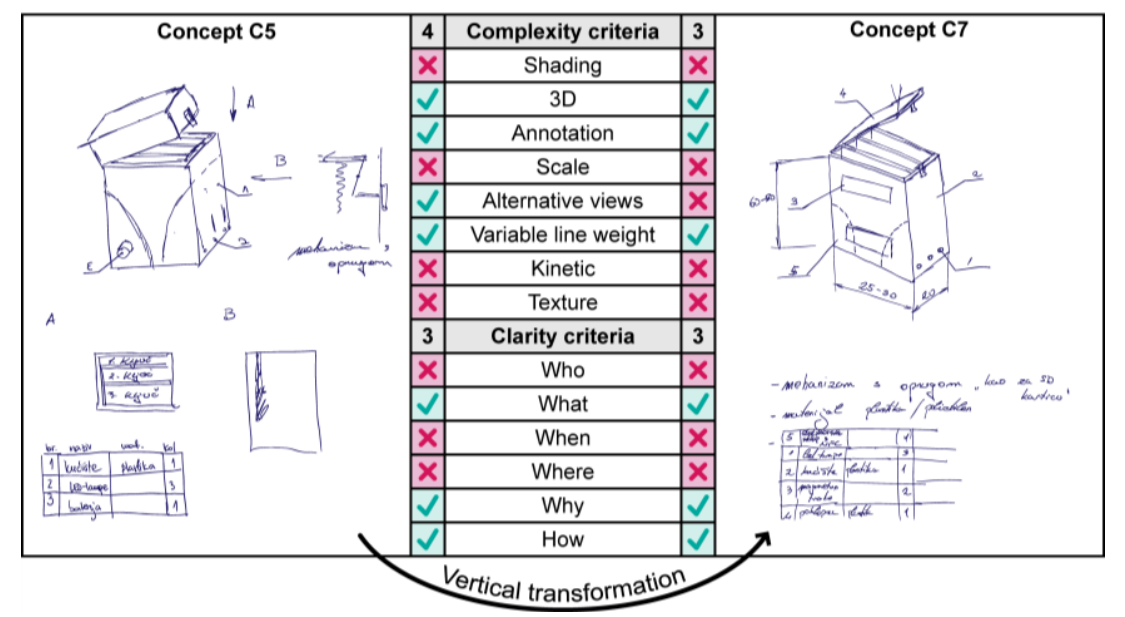

Figure 1. An example of complexity, clarity and transformation analysis for two concepts generated by Team 1 (based on Sevier et al., 2017)

\subsection{Sketching-related activity protocol coding}

As mentioned earlier, the coding scheme by Ariff et al. (2012) was selected as a basis for coding the sketching-related activities in the experiment sessions. The communication section of the designcommunication framework has been adapted for the purpose of this study. Namely, the "transferring" activity has been replaced with the "verbal explanation" activity due to different settings of the experiment itself. The original coding scheme (Ariff et al., 2012) was intended for teams that had the limitation of verbal communication. Therefore, there was a 'transferring' code that involved writing the conversation sentences and was used by teams that were not allowed to talk. In the experiment described in this paper, the teams did not have any restrictions, and the initial screening of the experiment records confirmed that 
such code was not needed and has thus been replaced with an equivalent code of verbal nature. The code is labelled "verbal explanation" and represents only the sketch-related verbalisation by team members. The differences between adapted and the original coding scheme (Ariff et al., 2012) are shown in Table 1. The adapted coding scheme has been used for identifying sketching-related activities, which included drawing (sketching), writing notes, and verbal referencing to sketches.

\section{Table 1. The differences of the coding scheme in relation to the original code scheme}

\begin{tabular}{lll}
\hline \multicolumn{1}{c}{ Activity } & \multicolumn{1}{c}{ Categorisation element } & \multicolumn{1}{c}{ Element } \\
\hline $\begin{array}{l}\text { Detailing: Extending / editing draft elements } \\
\text { through additional sketches or technical }\end{array}$ & $\begin{array}{l}\text { Technical elements: provide a thorough } \\
\text { technical understanding of the existing } \\
\text { elements }\end{array}$ & $\begin{array}{l}\text { Units / dimensions: value } \\
\text { and / or unit of technical } \\
\text { parameters such as length }\end{array}$ \\
\hline $\begin{array}{l}\text { Verbal explanation: Hand gestures and verbal } \\
\text { presentation i.e. sketches are explained by }\end{array}$ & & \\
$\begin{array}{l}\text { referring to sketches and clarifying the meaning } \\
\text { of sketch elements or sketch as a whole }\end{array}$ & & \\
\hline
\end{tabular}

When segmenting the protocol, the focus was on sketching and referring to sketches by determining team activities according to the coding scheme and participants in those activities. All identified segments are based on communication of team members by sketching and writing on paper and by referring to sketches and verbally explaining them. Each activity of a particular participant is associated with the given segment by specifying the following steps: (1) the beginning of the coding segment; (2) assigning of participants to a coded segment; (3) the duration or end of the coding segment; (4) assigning of code based on the coding scheme to the participant's activities. In the situation when multiple participants took part in the activities at the same time, each activity is associated with the segment and coded separately.

\section{RESULTS AND DISCUSSION}

The quantities of generated concepts and associated sketches during the analysed experiment sessions are listed in Table 2. The table shows the number of concepts per each team, including the final detailed concept for a given design task, and the number of drawn sketches for each of the concepts.

Table 2. Number of concepts and associated sketches generated by each team during the conceptual design sessions

\begin{tabular}{|c|c|c|c|c|c|c|c|c|c|c|c|c|}
\hline Team & C1 & $\overline{C 2}$ & C3 & $\overline{C 4}$ & C5 & C6 & C7 & C8 & C9 & $\mathbf{C 1 0}$ & Total & No. of concepts \\
\hline Team 1 & 3 & 1 & 6 & 4 & 4 & 0 & 1 & & & & 19 & 7 \\
\hline Team 2 & 1 & 5 & 0 & 1 & 6 & 5 & 4 & & & & 26 & 7 \\
\hline Team 3 & 1 & 5 & 1 & 3 & 1 & 2 & 2 & 2 & 10 & 4 & 31 & 10 \\
\hline Team 4 & 2 & 3 & 3 & 4 & 3 & & & & & & 15 & 5 \\
\hline Team 5 & 1 & 2 & 13 & 10 & 3 & & & & & & 29 & 5 \\
\hline
\end{tabular}

Among all analysed teams, Team 3 generated the largest number of concepts (10), each consisting of one or more sketches. In addition, Team 3 produced the highest number of individual sketches (31) which indicates the utmost importance of sketching as a communication medium throughout their conceptual design session. On the other hand, Teams 4 and 2 generated the lowest number of individual sketches (15 and 19, respectively). Team 2 even discussed one concept for which they didn't use drawing elements $(\mathrm{C} 1)$ since concept ideas were represented only with textual and without any pictorial description. In the further analysis, the text has been treated as a sketch element, but for this initial representation of results, it was not necessary to take it into consideration. Also, certain concepts ( $\mathrm{C} 6$ done by Team 1 and $\mathrm{C} 3$ done by Team 2) were related to neither one sketch because they were unfinished and, therefore, unusable for the analysis conducted in this study.

\subsection{Analysis of sketching outputs}

In terms of complexity, the evaluation method indicated that all concept sketches range between the levels 0 and 6 (out of 8), mostly fulfilling criteria of 3D Representation, Annotation, Alternative Views/Configuration and Variable Line Weight. Regarding clarity, prevalent concept sketch value was 3 (out of 6) mostly satisfying criteria of What, Why and How. The scores for the individual concepts are shown in Figure 2. When comparing assessed values among concept sketches for given conceptual design task, it can be easily perceived that clarity values oscillate between 2 and 3, while complexity values vary within a wider interval. These results point to the fact that teams on average put more emphasis on fundamental sketching and technical elements by addressing complexity criteria than embracing a broader understanding of the concept 
through a number of clarity criteria (Sevier et al., 2017). Still, a proper balance between complexity and clarity was established for most of the sketches as a consequence of their explanative nature (Kudrowitz et al., 2012).
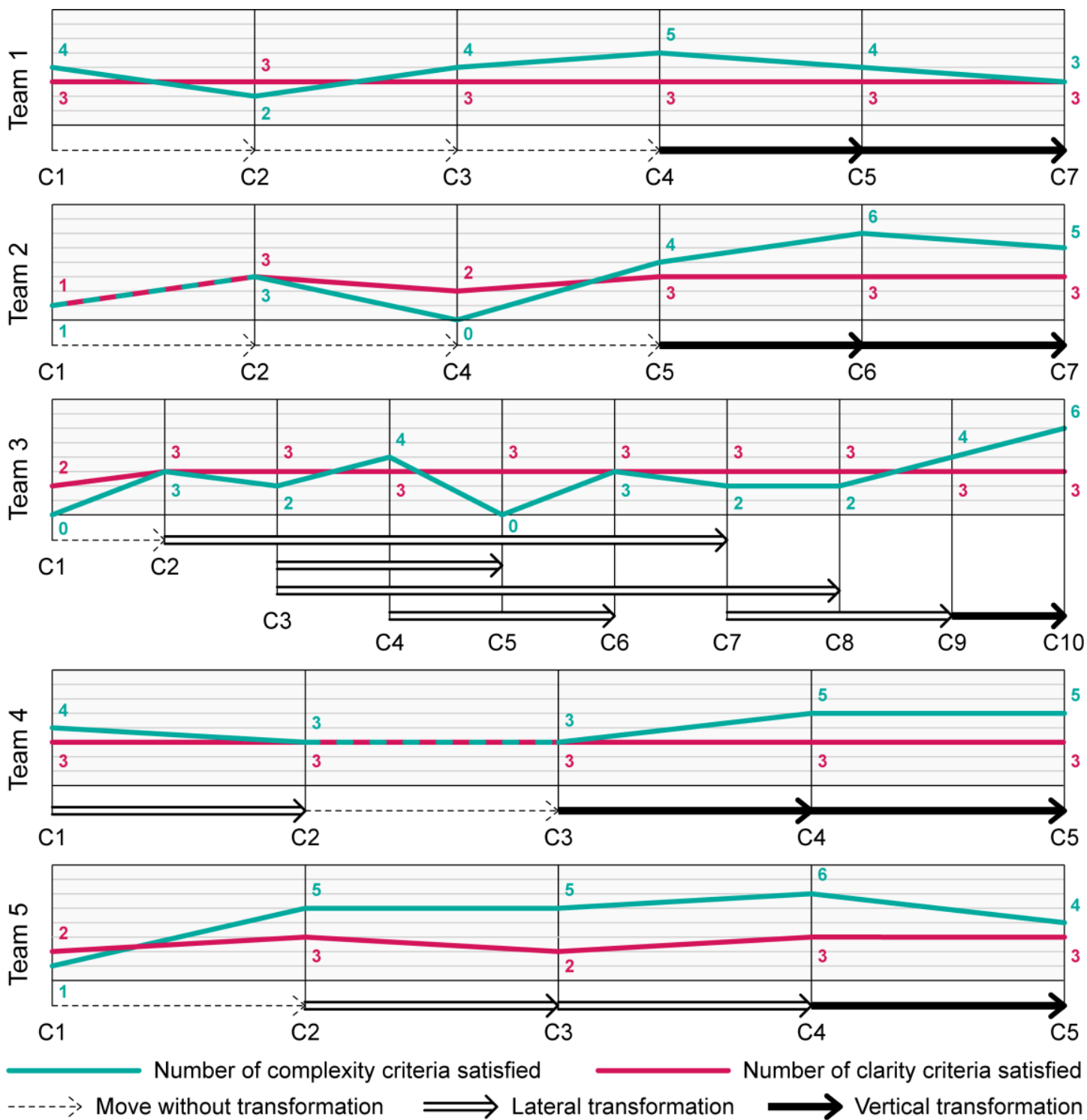

Figure 2. Sequences of concepts generated during team conceptual design sessions. Moves between the concepts are assigned with the type of sketch transformation, and the concepts are evaluated in terms of complexity and clarity

When analysing the dynamics of complexity values throughout sessions, results do not show a steady and continuous increase or decrease of these values. Teams used various approaches to develop the final concept while performing vertical and lateral transformations to switch to other concepts (Goel, 1995). At the beginning of the sessions, team members generated many different ideas without performing any transformations. Throughout the sessions, they commonly used lateral transformations to alter one concept into subsequent another. However, transformations to new concepts based on the principle of the already drawn, existing concept did not manifest itself in the complexity values. This may be again related to the trade-off between used criteria to maintain a sufficient level of sketch elaboration required to share ideas and concepts. Finally, when teams decided on the final concept, they used vertical transformations to further develop and detail certain concept based on the initial sketch. Therefore, lateral transformation, in case it happens, takes place during the generation phase, while the vertical transformation of sketches takes place during the decision-making and detailing phase of the final concept. The differences in the number of different transformations can partially be explained as a result of different types of associations appearing within the teams and due to the unidentified variations in experience background between the participants (see Yuan et al., 2018). 


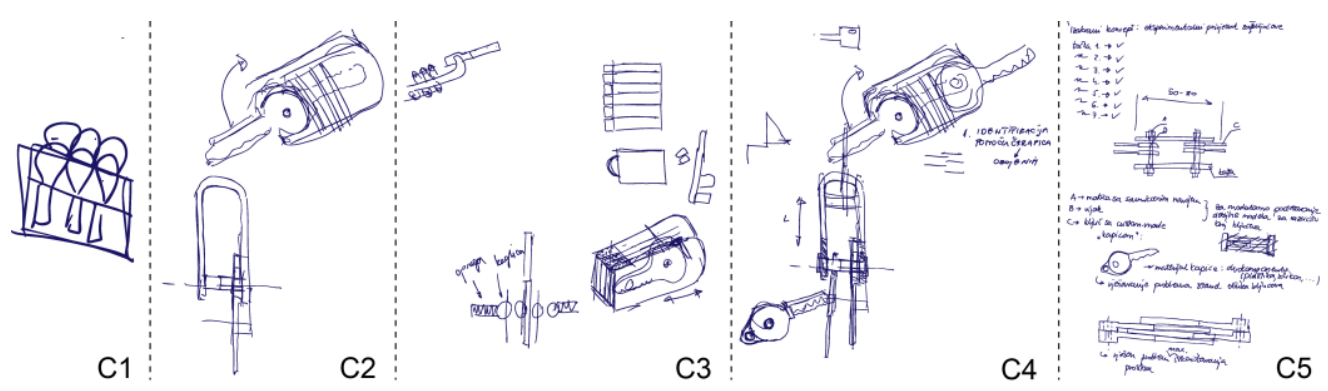

Figure 3. Sketches of concepts generated by Team 5. Concepts $C 3$ and C4 are the results of a lateral, and concept $C 5$ of a vertical sketch transformation

Concerning the number of sketches generated in relation to a single concept, it can be perceived that teams tend to increase the number before deciding on their final solution proposal. However, the reasons for the overall number of sketches created by a particular team can be sought in the corresponding sketching-related activities.

\subsection{Relating sketches to sketching-related activities}

Analysis of sketching-related activities and their relation to the developed concepts provides additional insights into the role of individual concepts and indicates the approach taken by teams along the session (Table 3). For that reason, the previously mentioned decrease in the number of sketches from the next-to-final to the final concept is also reflected in the number of associated sketching-related activities. To be more specific, for teams 2, 3, 4 and 5, the next-to-final concept is related to the highest number of sketching-related activities, with the only exception of Team 1. In general, towards the end of the conceptual design session, the number of activities related to individual concepts continuously increases.

Regarding the role of individual concept, it can be noticed that teams 1, 2 and 3 have the one concept that has a significantly higher number of related sketching activities $(55,103,114$ respectively) and dominates throughout the session, while team 5 has two of them. Team 4 has the most balanced distribution of sketching-related activities between concepts 3, 4 and 5 (between 19\% and 25\% of overall number of sketching-related activities for that team), but also the lowest total number of sketching-related activities, meaning that they less used sketches as a communication medium throughout the session compared to other teams. Also, this was further supported by the lowest number of concept sketches generated during the session.

\subsection{Analysis of sketching-related activity categories}

To better understand the role of sketching-related activities throughout the conceptual design session, the activities have been mapped on the four previously defined categories before being further analysed. The activities have been sorted and summed up for each team in Table 3. In addition, the table represents percentages for each coded activity category concerning the total number of coded segments (frequency distribution). Such detailed representation can explain the proportions of activities related to different concepts. Digging in deeper, dominating concepts developed in Teams 1, 2, 3 and 5, mostly owe their prevalence to high proportions of related Verbal explanation and Detailing activities. The only exception was Team 4, where Explaining activities have the highest proportion of coded segments during analysed conceptual design sessions. On the other hand, the Drawing activities are, on average, the least frequent with the proportion less or equal to $7 \%$ of coded segments. The low proportion of Drawing activities aligns with the findings reported by Ariff et al. (2012), although they appear continuously throughout the session and serve as a basis for the further development of the conceptual idea.

The protocol of Team 3 has the highest number of segments (266), in which verbal explanation activities dominate $(172,65 \%)$ followed by detailing $(65,24 \%)$, explaining $(19,7 \%)$ and, finally, drawing $(10,4 \%)$. While protocols of Teams 1,2 and 5 all follow a similar pattern, Team 4 exhibits a slightly different order of activities and significantly lower number of segments when compared to other teams (only 69). 
Table 3. Frequency distribution of sketch-related activity categories for each team

\begin{tabular}{|c|c|c|c|c|c|c|c|c|c|c|}
\hline \multirow{2}{*}{$\begin{array}{l}\text { Activity } \\
\text { Drawing }\end{array}$} & \multirow[t]{2}{*}{ Team } & Team 1 & \multicolumn{2}{|c|}{ Team 2} & \multicolumn{2}{|c|}{ Team 3} & \multicolumn{2}{|c|}{ Team 4} & \multicolumn{2}{|c|}{ Team 5} \\
\hline & & $7 \%$ & 6 & $3 \%$ & 10 & $4 \%$ & 5 & $7 \%$ & 8 & $4 \%$ \\
\hline Explaining & 33 & $20 \%$ & 15 & $7 \%$ & 19 & $7 \%$ & 30 & $44 \%$ & 17 & $9 \%$ \\
\hline Detailing & 27 & $17 \%$ & 48 & $23 \%$ & 65 & $24 \%$ & 11 & $16 \%$ & 58 & $32 \%$ \\
\hline Verbal explanation & 91 & $56 \%$ & 143 & $67 \%$ & 172 & $65 \%$ & 23 & $33 \%$ & 100 & $55 \%$ \\
\hline Total no. of segments & 163 & $100 \%$ & 212 & $100 \%$ & 266 & $100 \%$ & 69 & $100 \%$ & 183 & $100 \%$ \\
\hline
\end{tabular}

This revealed pattern can be explained through the associated sketch elements that were used as a sketching object during the session. To start with, the low number of Drawing segments stems from the fact that teams used associated sketch elements only when they began drawing a new sketch and then alternate other activities to visualise better or explain it. The proportions of Detailing and Explaining activities vary across teams, depending on their focus on additional supporting/technical sketch elements or graphical, numerical or textual explanation information. Finally, Verbal explanation activities were the most common since sketches often acted as stimuli for discussion and as a point of reference for elaborating ideas and thoughts of an individual team member. The comparison with the study performed by Ariff et al. (2012) revealed the broad alignment between results. However, when examining the one-directional transitions between Explaining, Detailing and Transferring, they were not confirmed in this study due to their consistent alternation along the sessions.

\subsection{Limitations and further work}

Although several claims have been made for the use of sketches in team conceptual design sessions, larger sample sizes are needed to validate and broaden the gathered insights. Moreover, future studies could also include the contextual dimension of sketching, that is the qualitative description of both the overall concepts sketches and the individual elements within these sketches. These qualitative descriptions should provide an additional perspective on the relation between the sketching-related activities, sketch elaboration and different aspects of the proposed solutions. In addition, the concepts generated as part of the presented empirical study have not been evaluated in terms of satisfying the design task requirements. Concept assessment must be performed to gain an understanding of what patterns of sketching activities and levels of sketch elaboration are likely to results in better concept designs. Finally, the presented results are based on short-term empirical study, which included the hour-long team sessions only. Moreover, due to the setup of the experiment, only teams of three members have been investigated, and one person could sketch simultaneously. Sessions of different durations, with varying sizes of teams and sketching equipment allocation, could provide additional insights into the interaction of sketching-related activities and the level of elaboration of the sketching outputs.

\section{CONCLUSION}

The presented empirical study revealed both the frequencies of different sketching-related activities during team conceptual design sessions and the associated levels of elaboration for each of the sketching outputs in the form of concept drawings. The results show that teams used various approaches regarding performing vertical and lateral transformations to switch between concept sketches before the final concept proposal was produced. Nonetheless, teams share a pattern of developing solutions without transformations or using lateral transformations within the first part of the sessions (generation phase) and using vertical transformations to produce final concepts towards the end of the sessions (decision-making and detailing phase). Moreover, the results confirm that teams put more emphasis on fundamental sketching and technical elements by addressing complexity criteria, compared to embracing a broader understanding of the concept through a number of clarity criteria. In terms of the sketch-related activities, it can be concluded that teams used associated sketch elements to start drawing new sketches and then alternate other activities for the sake of better elaboration and understanding. Interestingly, the verbal explanation was the most common sketch-related activity, as sketches provided stimuli for both the team discussion and for elaborating ideas and thoughts of the individual members.

\section{REFERENCES}

Agogino, A., Song, S. and Hey, J. (2006), "Triangulation of Indicators of Successful Student Design Teams", International Journal of Engineering Education, Vol. 22 No. 3, pp. 617-625. 
Besemer, S.P. (1998), "Creative product analysis matrix: Testing the model structure and a comparison among products - Three novel chairs”, Creativity Research Journal, Vol. 11 No. 4, pp. 333-346. https://doi.org/10.1207/s15326934crj1104_7

Chen, H.-H., You, M. and Lee, C.-F. (2003), "The sketch in industrial design process", Proceedings of the 6th Asian Design Conference: Integration of Knowledge, Kansei, and Industrial Power, Tsukuba, Japan, October 14-17, 2003.

Dean, D.L., Hender, J.M., Rodgers, T.L. and Santanen, E. (2006), "Identifying good ideas: constructs and scales for idea evaluation", Journal of Association for Information Systems, Vol. 7 No. 10, pp. 646-699.

Dorst, K. and Dijkhuis, J. (1995), “Comparing paradigms for describing design activity”, Design Studies, Vol. 16 No. 2, pp. 261-274. https://doi.org/10.1016/0142-694X(94)00012-3.

Eris, O., Martelaro, N. and Badke-Schaub, P. (2014), "A comparative analysis of multimodal communication during design sketching in co-located and distributed environments”, Design Studies, Vol. 35 No. 6, pp. 559-592. https://doi.org/10.1016/j.destud.2014.04.002

Fish, J. and Scrivener, S. (1990), “Amplifying the mind's eye: Sketching and visual cognition”, Leonardo, Vol. 23 No. 1, pp. 117-126. https://doi.org/10.2307/1578475

Gero, J.S. (1990), "Design Prototypes: A Knowledge Representation Schema for Design”, AI Magazine, Vol. 11 No. 4, pp. 26-36. https://doi.org/10.1609/aimag.v11i4.854

Goel, V. (1995), Sketches of Thought, MIT Press.

Goldschmidt, G. (1991), “The Dialectics of Sketching”, Creativity Research Journal, Vol. 4 No. 2, pp. $123-143$. https://doi.org/10.1080/10400419109534381

Heiser, J., Tversky, B. and Silverman, M.I.A. (2004), "Sketches for and from collaboration", Preprints of the 3rd International Conference on Visual and Spatial Reasoning in Design, Massachusetts Institute of Technology, Cambridge, USA, July 22-23, 2004, pp. 69-78.

Kudrowitz, B., Te, P. and Wallace, D. (2012), "The influence of sketch quality on perception of product-idea creativity", Artificial Intelligence for Engineering Design, Analysis and Manufacturing, Vol. 26 No. 3, pp. 267-279. https://doi.org/10.1017/S0890060412000145

van der Lugt, R. (2002), "Brainsketching and how it differs from brainstorming", Creativity and Innovation Management, Vol. 11 No. 1, pp. 43-54. https://doi.org/10.1111/1467-8691.00235

Martinec, T., Horvat, N., Škec, S. and Štorga, M. (2018), "Verbal engagement in teams solving a conceptual design task", Proceedings of the DESIGN 2018 15th International Design Conference, pp. 2075-2086. https://doi.org/10.21278/idc.2018.0540

Neill, T.M., Gero, J.S. and Warren, J. (1998), "Understanding conceptual electronic design using protocol analysis", Research in Engineering Design, Vol. 10 No. 3, pp. 129-140. https://doi.org/10.1007/BF01607155

Nik Ahmad Ariff, N.S., Badke-Schaub, P., Eris, O. and Suib, S.S.S. (2012), “A framework for reaching common understanding during sketching in design teams", Proceedings of the 12th International Design Conference (DESIGN 2012), Dubrovnik, Croatia, May 21-24, 2012, pp. 1525-1533.

Purcell, A.T. and Gero, J.S. (1998), "Drawings and the design process: A review of protocol studies in design and other disciplines and related research in cognitive psychology", Design Studies, Vol. 19 No. 4, pp. 389-430. https://doi.org/10.1016/S0142-694X(98)00015-5

Rodgers, P.A., Green, G. and McGown, A. (2000), "Using concept sketches to track design progress”, Design Studies, Vol. 21 No. 5, pp. 451-464. https://doi.org/10.1016/S0142-694X(00)00018-1

Sachse, P., Hacker, W. and Leinert, S. (2004), "External thought - Does sketching assist problem analysis?", Applied Cognitive Psychology, Vol. 18 No. 4, pp. 415-425. https://doi.org/10.1002/acp.992

Scrivener, S.A.R., Ball, L.J. and Tseng, W. (2000), "Uncertainty and sketching behaviour", Design Studies, Vol. 21 No. 5, pp. 465-481. https://doi.org/10.1016/S0142-694X(00)00019-3

Sevier, D.C., Jablokow, K., McKilligan, S., Daly, S.R., Baker, I.N. and Silk, E.M. (2017), "Towards the Development of an Elaboration Metric for Concept Sketches", Proceedings of the ASME 2017 IDETC-CIE Conference, Ohio, USA, August 6-9, 2017, p. V003T04A006.

Suwa, M., Purcell, T. and Gero, J. (1998), "Macroscopic analysis of design processes based on a scheme for coding designers' cognitive actions”, Design Studies, Vol. 19 No. 4, pp. 455-483. https://doi.org/10.1016/S0142-694X(98)00016-7

Ullman, D.G., Wood, S. and Craig, D. (1990), "The importance of drawing in the mechanical design process", Computers and Graphics, Vol. 14 No. 2, pp. 263-274. https://doi.org/10.1016/0097-8493(90)90037-X

Yuan, P., Li, Y., Chen, J., Xiong, Y. and Liu, L. (2018), "The importance of drawing in the mechanical design process", Journal of Mechanical Design, Vol. 140 No. 10, p. 101102. https://doi.org/10.1115/1.4040627

\section{ACKNOWLEDGMENTS}

This paper reports on work funded by the Croatian Science Foundation project IP-2018-01-7269: Team Adaptability for Innovation-Oriented Product Development - TAIDE (http://www.taide.org). 